Carbocation Chemistry; edited by G. A. Olah and G. K. Surya Prakash; John Wiley \& Sons: Hoboken, 2004, hardcover, 393 pp, US\$ 99.95, ISBN 0-471-28490-4

\section{Chemical positivism}

Carbocations are one of the most important groups of reactive intermediates that are formed during the course of a reaction and which usually have only a fleeting existence. After carbocations were found to be real entities in the early days of the 20th century, the following decades saw researchers probe into their structures and chemical reactivities in ever more detail. Many of the pioneers who helped develop this field of organic chemistry have contributed to this handy volume on the topic.

The idea for this book goes back to a symposium in 2001 on the occasion of ' 100 years of carbocations'. The fourteen chapters of the volume spawned by that conference cover a broad range of topics, tied together by the common denominator of carbocations. The book opens with a very neat, easy-to-read account of the history and development of the entire field. George Olah, one of the editors and a towering figure during the blossoming renaissance of the field during the second half of the 20th century, follows with a more detailed account of the evolution of carbocation research that features chemistry over history and which is tinted with a distinctly personal flavour. In a few of the remaining chapters, a personal touch occasionally appears, but most contributions are very sober accounts in the familiar form of major review articles. All chapters have been written with great authority. The editors have opted for a very liberal mode of editing. While this gives the contributing authors a maximum of freedom, it inevitably fires back in one way or another. Some of the authors are apparently fighting long-standing battles with the English language; so much so in some cases that the readability is markedly decreased and the reading of an otherwise scientifically stimulating contribution becomes quite a chore.
While carbocations are normally short-lived intermediates, interest in more persistent forms that would allow for easier study go back as far as Baeyer's discovery of 'carbonium salts', the very discovery that marks the date of birth of carbocation research. Two chapters specifically address the challenge of stabilized, long-lived carbocations.

Two other contributions address the topic of carbocations in organometallic compounds. Strong acids and superacids have proved indispensable for carbocation research. In due course, they appear in practically every chapter of the book, but chapters twelve and fourteen give credit to these remarkable reagents by featuring them exclusively. Due to the highly dynamic character of carbocations in general, most contributions have a major focus on reactions and reaction dynamics rather than structure.

Carbocation Chemistry is a valuable state-of-the-art report of an important field of basic organic chemistry. While it does not neglect the historical perspective, it emphasizes modern developments, and so updates earlier monographs on this topic. For institutional libraries of chemical institutes, it will be a required purchase. Although it does not quite meet the criteria to qualify as a bargain, for a specialized monograph, the prize is still affordable even for interested individuals (postdoc level and up). For laboratories actively engaged in research projects which involve carbocations, it is a must-have that will serve as a thorough guide for postgraduates embarking on a career in physical organic chemistry.

Thomas Lazar (retired) The Max Planck Institute for Biophysical Chemistry, Göttingen, Germany
SYNTHESIS 2005, No. 20, pp 3672-3672

Advanced online publication: 07.12.2005

Art ID: B12005SS

(C) Georg Thieme Verlag Stuttgart · New York 